\title{
Occurrence of Headache with and without Pillow Cushion in Supine Position after Spinal Anesthesia
}

\author{
Maharjan D ${ }^{1}$ \\ ${ }^{1}$ Department of Anesthesiology, Kathmandu Model Hospital, Kathmandu, Nepal
}

Received: 5-Mar-2017; Accepted: 30-May-2017

Aims: This study was designed to define the role of position in the occurrence of postdural puncture headache (PDPH) after spinal anesthesia. Methods: This is a comparative study of 69 patients in each group with pillow cushion and without during spinal anesthesia
in supine position for the patients scheduled for low abdominal and pelvic surgeries.

Results: Patients in both groups experienced headache (but not PDPH) after surgery with slightly higher ( $\mathrm{n}=13$, $18.84 \%$ ) rate in strict supine position than with head rest using a pillow cushion $(\mathrm{n}=11,15.94 \%)$. This was not statistically significant $(\mathrm{p}=0.65)$.

Conclusions: Supine position with a pillow cushion in the immediate post-spinal period caused a non-significant decrease in the incidence of headache and a significant increase in comfort. This study could not conclude whether a pillow cushion affects the occurrence of PDPH as there were no cases of PDPH in either group.

Keywords: post-dural puncture headache; spinal anesthesia; supine; pillow cushion

DOI: http://dx.doi.org/10.3126/njog.v12i1.18983

\section{INTRODUCTION}

Post-dural puncture headache (PDPH) is as high as $25 \%$ in patients due to technique-related complications of spinal anaesthesia. ${ }^{1}$ The hallmark of such condition is the occurrence of fronto-occipital headache that is aggravated by head elevation in the upright position but improves upon returning to the supine position. ${ }^{1-3}$ The headache is believed to result from decrease in intracranial pressure from loss of cerebrospinal fluid (CSF) through the dural hole, decreasing buoyant support for the brain. In the upright position, the brain sags into the cranial vault, putting traction on pain sensitive structures causing headache. Moreover, decreased CSF results in a compensatory increase in cerebral blood volume causing vasodilatation which is responsible for the headache. ${ }^{4}$ Most anesthesiologists recommend that patients should lie flat in bed for several hours after the procedure is performed. This is believed to decrease CSF hydrostatic pressure that may affect the rate of CSF leak from the dural puncture.

\section{CORRESPONDENCE}

Dr Devendra Maharjan

Department of Anesthesiology,

Kathmandu Model Hospital, Kathmandu, Nepal

Phone: +977-9851156567, Email: mahdevendra@yahoo.com
This study was designed because there are very few studies that have focused on the importance of position, its role and significance in the occurrence of PDPH.

\section{METHODS}

This study was conducted among 140 surgical patients in the Ilocos Training and Regional Medical Center, San Fernando City, Philippines from July 2011 to March 2012. They were scheduled for low abdominal surgical procedures including obstetricgynecologic and surgical procedures. Case enrollment was randomized by a shuffled deck of cards (e.g., even - odd) after informed consent was obtained. Visual Analogue Scale (VAS) was explained to all patients during the preoperative visit. Patients with known psychiatric disorder, bronchial asthma, hypertension, diabetes, heart disease or American society of anesthesiologists (ASA) status III or above, history of PDPH, chronic back pain or trauma, headache, migraine, vertigo, sinusitis, chronic use of analgesics, those who received two or more spinal tap attempts or experienced paraesthesia during the spinal tap procedure and age below 18 years and above 60 years were excluded from this study. The patients were divided into two groups randomly, each group consisting of 70 patients but one patient in each group was excluded because of a double tap. Group 
A belonged to the supine group without a pillow cushion and group B belonged to the supine with a pillow cushion.

On arrival to the operating room, all patients were pre-medicated with an anxiolytic dose of midazolam 1-2mg IV while in the waiting area. Routine monitoring was connected and supplemental oxygen administered. All patients were pre-hydrated with lactated ringer's $10 \mathrm{ml}$ per kilogram bodyweight. Each subject received subarachnoid block with bupivacaine $0.5 \%$ heavy $(2-3.5 \mathrm{ml}$, depending on the type of surgery) using a cutting spinal needle (Quincke) of gauge 25 at L3-L4 or L4-L5 interspaces in the sitting position. After assessing the desired dermatomal level of blockade, patients were sedated with intravenous nalbuphine $5 \mathrm{mg}$ plus midazolam $1 \mathrm{mg}$. Vital signs (blood pressure, pulse rate and respiratory rate) were monitored every five minutes.

Patients in group A were required to assume strict supine position, which was in the horizontal position without a pillow cushion for eight hours whilst those in group B were required to maintain head rest using a standard pillow cushion immediately after the subarachnoid block was performed. Nalbuphine 5 to $10 \mathrm{mg}$ IV every four hours and ketorolac $15-30 \mathrm{mg}$ every six hours or mefenamic acid $500 \mathrm{mg}$ every six hours orally were delivered as post- operative pain killers. All patients were examined after 24 hours. Patients who complained of headache post-operatively were assessed using the pain diary to confirm the diagnosis of PDPH. Necessary interventions were done to address patients' concerns. All subjects were followed up through phone calls up to two weeks after hospital discharge and were advised to report to the hospital for any untoward signs and symptoms. Results were analyzed using a two-tailed t-test and a p-value of $<0.05$ was considered statistically significant.

\section{RESULTS}

Initially 140 patients were enrolled in the study. Two patients were excluded since both received two attempts at subarachnoid block during induction of anesthesia. Both supine and supine with a pillow cushion groups consisted of 69 subjects each for analysis. Patients in both groups experienced headache after surgery with group A having slightly higher occurrence than group B (Table 1). Where, the $\mathrm{p}$-value was 0.65 . The result was not significant at $\mathrm{p}$ $<.05$.
Table1. Summary of patients who experienced headache after surgery $(n=138)$.

\begin{tabular}{|llll|}
\hline Headache & Yes & No & Total \\
\hline $\begin{array}{l}\text { Group A } \\
\text { (without } \\
\text { pillow) }\end{array}$ & $13(18.84 \%)$ & $56(81.16 \%)$ & $69(100 \%)$ \\
$\begin{array}{l}\text { Group } \\
\text { B (with } \\
\text { pillow) }\end{array}$ & $11(15.94 \%)$ & $58(84.64 \%)$ & $69(100 \%)$ \\
Total & $24(17.40 \%)$ & $114(82.60 \%)$ & $138(100 \%)$ \\
\hline
\end{tabular}

The $p$-value is 0.65 . The result is not significant at $p$ $<0.05$.

As to the description of headache, The majority of subjects in both groups described their headache as a dull, throbbing or pulsating pain and localized to the frontal, temporal and occipital regions (Tables 2 and 3).

Table 2: Description of headache in both groups $(n=24)$

\begin{tabular}{|lll|}
\hline Description & Group A & Group B \\
\hline Throbbing, pulsating & $4(30.77 \%)$ & $1(9.09 \%)$ \\
Dull pain & $7(53.85 \%)$ & $6(54.54 \%)$ \\
Squeezing, spastic & 0 & 0 \\
Continuous & 0 & $1(9.09 \%)$ \\
Intermittent & $2(15.38 \%)$ & $3(27.27 \%)$ \\
Total & $13(100 \%)$ & $11(100 \%)$ \\
\hline
\end{tabular}

Table 3: Location of headache in both groups $(n=24)$

\begin{tabular}{|lll|}
\hline Location & Group A & Group B \\
\hline Frontal & $6(46.15 \%)$ & $5(45.45 \%)$ \\
Temporal & $4(30.77 \%)$ & $3(27.27 \%)$ \\
Vertex & 0 & 0 \\
Nuchal & 0 & 0 \\
Generalized & $1(7.69 \%)$ & $3(27.27 \%)$ \\
Retro-orbital & 0 & 0 \\
Occipital & $2(15.35 \%)$ & 0 \\
Total & $13(100 \%)$ & $11(100 \%)$ \\
\hline
\end{tabular}

The results showed that neither group experienced very mild discomfort, (that is VAS scores 1-2), nor severe debilitating headache (that is VAS scores from 7-10). With this result, it showed that there was no significant difference in the severity or intensity of post-operative headache among patients in both groups (Table 4).

Table 4: Severity of headache of both groups using the VAS $(n=24)$

\begin{tabular}{|lll|}
\hline VAS Score & Group A & Group B \\
\hline $1-2$ & 0 & 0 \\
$3-4$ & $7(53.85 \%)$ & $7(63.64 \%)$ \\
$5-6$ & $6(46.15 \%)$ & $4(36.36 \%)$ \\
Total & $13(100 \%)$ & $11(100 \%)$ \\
\hline
\end{tabular}

As evident in table 5, neither group had headache associated with body posture or position. Seven 
patients (out of $13(53.85 \%)$ in group A had postoperative headache related to head movements alone while the remaining six patients (46.15\%) had continuous in both upright and flat positions. In group B, however, 10 patients $(90.91 \%)$ had continuous headache while only one patient $(9.09 \%)$ had a post-operative headache related to head movements alone. These results therefore, show that both groups suffered from headache that was not position related and therefore, not PDPH.

Table 5: Association of headache with body position or posture $(\mathrm{n}=24)$

\begin{tabular}{|lll|}
\hline Description & Group A & Group B \\
\hline $\begin{array}{l}\text { Aggravated by assuming } \\
\text { upright position }\end{array}$ & 0 & 0 \\
$\begin{array}{l}\text { Relieved by lying flat on } \\
\text { bed }\end{array}$ & 0 & 0 \\
$\begin{array}{l}\text { Exacerbated by head } \\
\text { movement alone }\end{array}$ & $7(53.85 \%)$ & $1(9.09 \%)$ \\
$\begin{array}{l}\text { Continuous in both upright } \\
\text { and flat positions }\end{array}$ & $6(46.15 \%)$ & $10(90.91 \%)$ \\
Total & $13(100 \%)$ & $11(100 \%)$ \\
\hline
\end{tabular}

Table 6 shows that, 31 (44.92\%) out of 69 patients of group A described some of their post-operative concerns as follows: neck pain/ stiffness, generalized musculoskeletal back pain and nausea. None had vomiting and the rest of the patients (38/69 or $55.07 \%$ ) were comfortable during their entire postoperative course. Conversely, in group B, only 13 patients $(18.84 \%)$ had other concerns postoperatively, which as follows: neck pain/stiffness, generalized musculoskeletal back pain and nausea. It is striking to recognize that in group B, only two patients (2.9\%) had neck pain/ stiffness which is $13.04 \%$ lower compared to Group A $(n=11,15.94 \%)$. Moreover, only six patients $(8.7 \%)$ in group B had generalized musculoskeletal back pain as compared to 15 patients $(21.74 \%)$ in group A. None had episodes of vomiting; 56 out of 69 patients $(81.16 \%)$ of group B were comfortable throughout their post-operative course as compared to 38 out of 69 patients $(55.07 \%)$ of Group B.

Table 6: Post-operative concerns of both groups A and $B(n=69)$.

\begin{tabular}{|lll|}
\hline Concerns & Group A & Group B \\
\hline $\begin{array}{l}\text { Neck pain and stiffness } \\
\text { Generalized }\end{array}$ & $11(15.94 \%)$ & $2(2.9 \%)$ \\
musculoskeletal back pain & $15(21.74 \%)$ & $6(8.69 \%)$ \\
Nausea & $5(7.25 \%)$ & $5(7.25 \%)$ \\
\hline
\end{tabular}

\begin{tabular}{|lll|}
\hline Vomiting & 0 & 0 \\
Others & 0 & 0 \\
None & $38(55.07 \%)$ & $56(81.16 \%)$ \\
Total & $69(100 \%)$ & $69(100 \%)$ \\
\hline
\end{tabular}

\section{DISCUSSION}

It was in the late 1800 s that spinal anesthesia (SA) was developed. Bier believed that the headache was attributed to loss of cerebrospinal fluid. ${ }^{1,3}$ By the early 1900 s, numerous reports stated that $50 \%$ of subjects experienced headache after receiving $\mathrm{SA}$ which at that time was said to resolve in 24 hours. $^{4}$

Light and microscopic studies of human dura mater showed that orienting the spinal needle at right angles to the parallel fibers would cut the fibers under tension causing it more further to retract and increase the dimensions of dural perforation increasing the likelihood of PDPH..$^{2-4}$ Barash et al. stated that remaining supine after meningeal puncture does not decrease the incidence of PDPH. ${ }^{1}$

Kane et $\mathrm{al}^{5}$ also reported in his research results that PDPH incidence is not influenced by putting the patient in a supine position with a pillow cushion, rather it improves patient comfort after surgery, which, were very similar to the findings in this study.

Previous studies have failed to find a significant correlation between the number of dural punctures and the incidence of PDPH, questioning the hypothesis that leakage of the CSF through the dural tear is the cause of PDPH. Seeberger et $\mathrm{al}^{6}$ re-examined whether repeated dural punctures increase the incidence of PDPH by analysing prospectively collected data on 8034 spinal anesthetics. They found that repeated dural punctures significantly increased the incidence of PDPH. It was concluded that increased risk of PDPH is a disadvantage of performing a second subarachnoid injection of local anaesthetics after failed SA. Moreover, this result suggests that leakage of CSF through the dural tear is the most plausible cause of PDPH.

Hafer et al, ${ }^{7}$ showed that post-operative recumbence or immobilization did not reduce the risk of PDPH and that the duration of strict bed rest did not influence the development of PDPH.With these reports, the traditional practice of having patients strictly in a supine position without a pillow cushion can be reviewed to improve patient comfort. This study was conducted to help establish or identify the role of position in the occurrence of PDPH with the hope 
that patients' post-operative recovery conditions can be improved.

In this research, all patients underwent low abdominal surgery under SA. Results showed that patients experienced ordinary non-posture related headache and none suffered posture-related headache that clearly defines PDPH. This result can therefore, strengthen the contention that position does not influence the incidence and occurrence of PDPH. Moreover, patients in the supine position with a pillow cushion after SA had decreased intensity of ordinary non-posture related headache as supported by the VAS scores results. They also had decreased incidence of other post-operative related concerns such as nausea, vomiting, neck pain and musculoskeletal back pain. The majority of subjects in group B did not complain of any post-operative discomfort. However, in this research, intraoperative and post-operative fluids were almost similar in volume in both the groups but we did not analyse. Other causes of headache after SA are paralysis of cranial nerves, chemical and infective meningitis but in this study, clinically, there were no signs and symptoms of these conditions.

\section{CONCLUSIONS}

Supine position with a pillow cushion in the immediate after SA caused a non significant decrease in the incidence of headache among patients undergoing low abdominal surgery. However, improved patient comfort was found by reducing the intensity of ordinary not posture related headache and reducing incidence of other post-operative concerns such as neck pain and stiffness, and generalized musculoskeletal back pain.

However, this study could not conclude whether a pillow cushion affects the occurrence of PDPH as there were no cases of PDPH in either group.

More studies are required to support the proposition that a pillow cushion is also safe for patients undergoing abdominal surgeries in the immediate post-spinal period and further studies should be conducted to strengthen the findings of this study in order to help contribute in establishing the exact role of position in the occurrence of PDPH.

\section{REFERENCES}

1. Barash PG, Cullen BF, Stoelting RK, Cahalan MK, Stock MC.Clinical Anesthesia, $6^{\text {th }}$ ed. Philadelphia, USA: Lippincott Williams and Wilkins.2009;947-8.

2. Morgan GE, Mikhail MS, Murray MJ. Clinical Anesthesiology. $4^{\text {th }}$ ed.New York, USA: McGraw-Hill Companies. 2006;319.

3. Miller RD, Eriksson LI, Fleisher LA, Wiener-Kronish JP, Young WL. Anesthesia, $7^{\text {th }}$ ed. Part A, Volume 2. Philadelphia: Churchill livingstone Elsevier.2010;1626-7.
4. Tumbull DK, Shepherd DB. Post $\square$ dural puncture headache pathogenesis, prevention and treatment. $\mathrm{Br} \mathrm{J}$ Anaesth. 2003;91:718-29.

5. Kane WJ, Peilan G. Intraoperative to pillow cushions and lumbar epidural anesthesia on post-operative headache impact. Shandong Medicine. 2003;43(33):47-8.

6. Seeberger MD, Kaufmann M, Starder S. Repeated dural punctures increase the incidence of post-dural puncture headache. Anaesth Analg. 1996;82(2):302-5.

7. Hafer J, Rupp D, Wollbruck M. The effect of needle type and immobilization on post-spinal headache. Anaesthesist 1997;46:860-6. 